\title{
Proteomic profiling of a fast neutron induced soybean mutant unveiled pathways associated with increased seed protein content
}

Nazrul Islam¹, Hari B. Krishnan² and Savithiry Natarajan ${ }^{1}$

${ }^{1}$ Soybean Genomics and Improvement Laboratory, USDA-ARS, MD.20705, USA 2Plant Genetics Research Unit, USDA-ARS, University of Missouri, Columbia, MO. 65211, USA.

Supporting Information (S)

Table S1. Total protein identified with at least 1 significant spectrum and their relative abundance. WT_1, wild rep1; WT_2, wild rep2; WT_3, wild rep3; 3_1, mutant rep1; 3_2, mutant rep2; 3_3, mutant rep3.

Table S2. Total protein identified with more than 1 significant spectrum and their normalized abundance. WT_1, wild rep1; WT_2, wild rep2; WT_3, wild rep3; 3_1, mutant rep1; 3_2, mutant rep2; 3_3, mutant rep3.

Table S3: Number of proteins that were up-regulated along with their peptide spectrum matches and p-values. WT_1, wild rep1; WT_2, wild rep2; WT_3, wild rep3; 3_1, mutant rep1; 3_2, mutant rep2; 3_3, mutant rep3.

Table S4: Number of proteins that showed significantly lower abundant along with their peptide spectrum matches and p-values. WT_1, wild rep1; WT_2, wild rep2; WT_3, wild rep3; 3_1, mutant rep1; 3_2, mutant rep2; 3_3, mutant rep3.

Table S5: Number of proteins that showed no significant difference along with their peptide spectrum matches and p-values. WT_1, wild rep1; WT_2, wild rep2; WT_3, wild rep3; 3_1, mutant rep1; 3_2, mutant rep2; 3_3, mutant rep3.

Figure S1. Gene Ontology terms of the lower abundant proteins analyzed by AgriGO (http://bioinfo.cau.edu.cn/agriGO/search.php

Figure S2: Protein associated with 29 ribosomal units increased significantly, of which 16 belongs to large ribosomal subunits and 13 to the small subunits. Red indicates higher abundance.

Figure S3: Mapping of ER proteins, Red indicates higher abundance and yellow indicates lower abundance. 


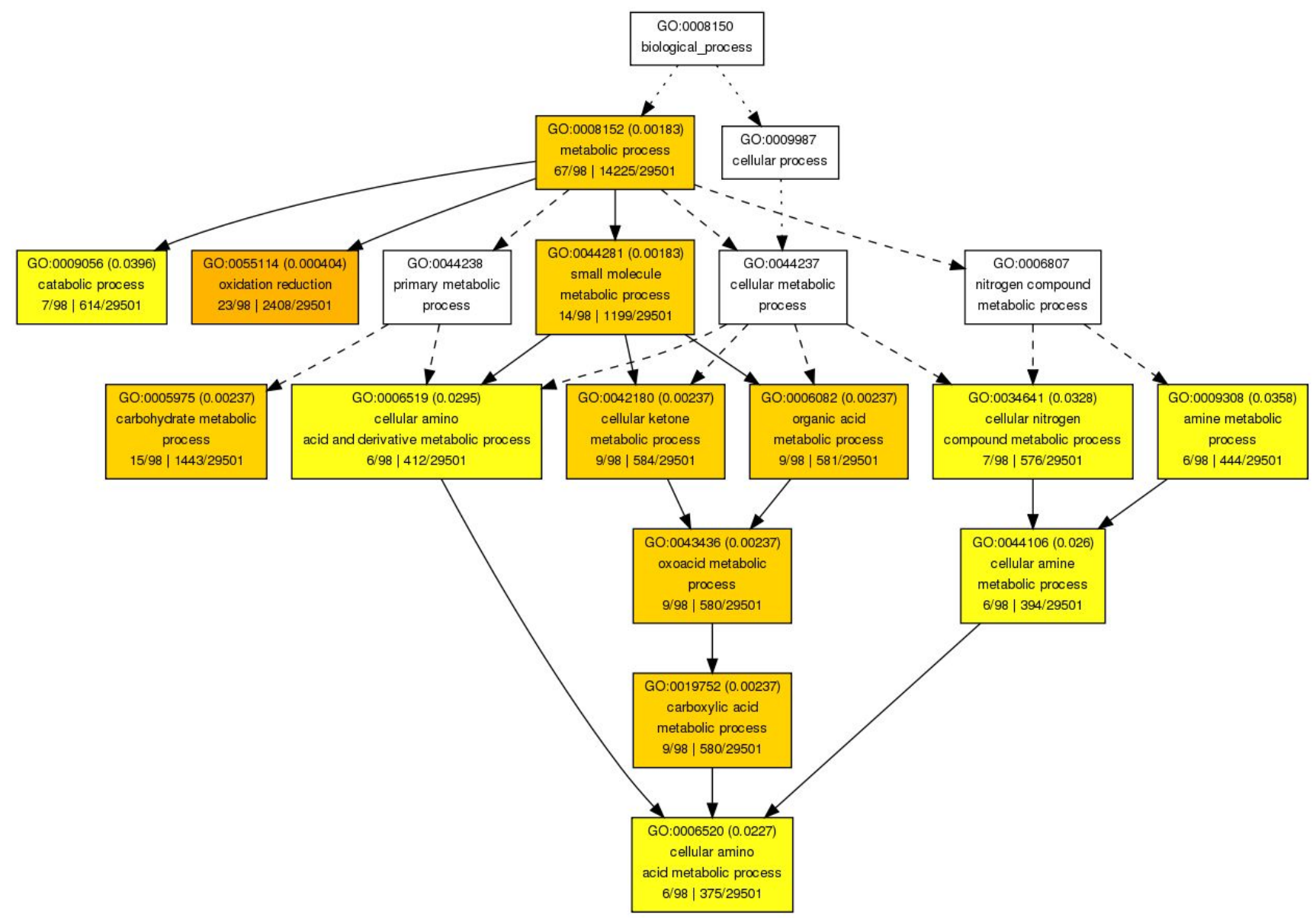

Figure S1. Gene Ontology terms of the lower abundant proteins analyzed by AgriGO (http://bioinfo.cau.edu.cn/agriGO/search.php 


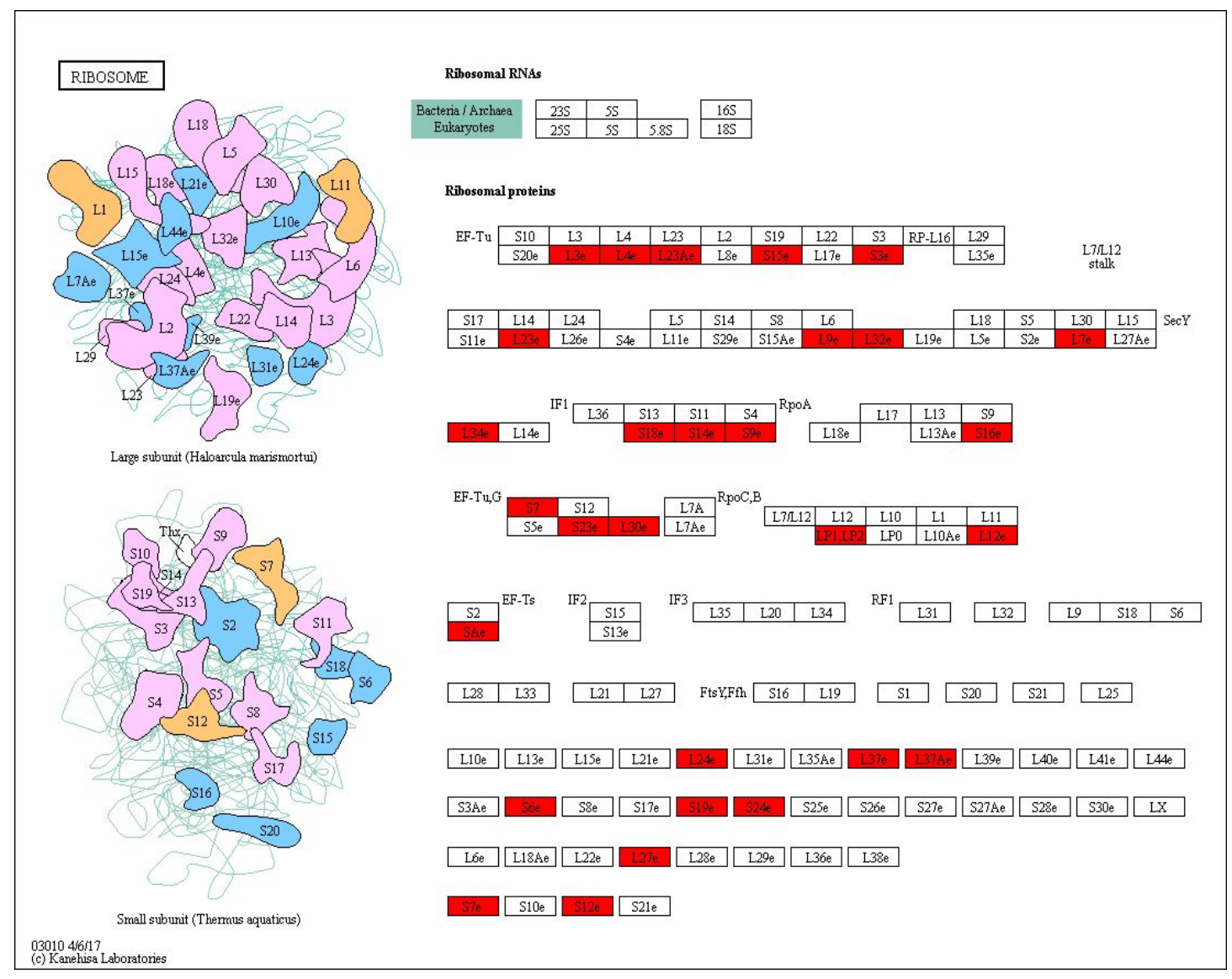

Figure S2: Protein associated with 29 ribosomal units increased significantly, of which 16 belongs to large ribosomal subunits and 13 to the small subunits. Red indicates higher abundance. 


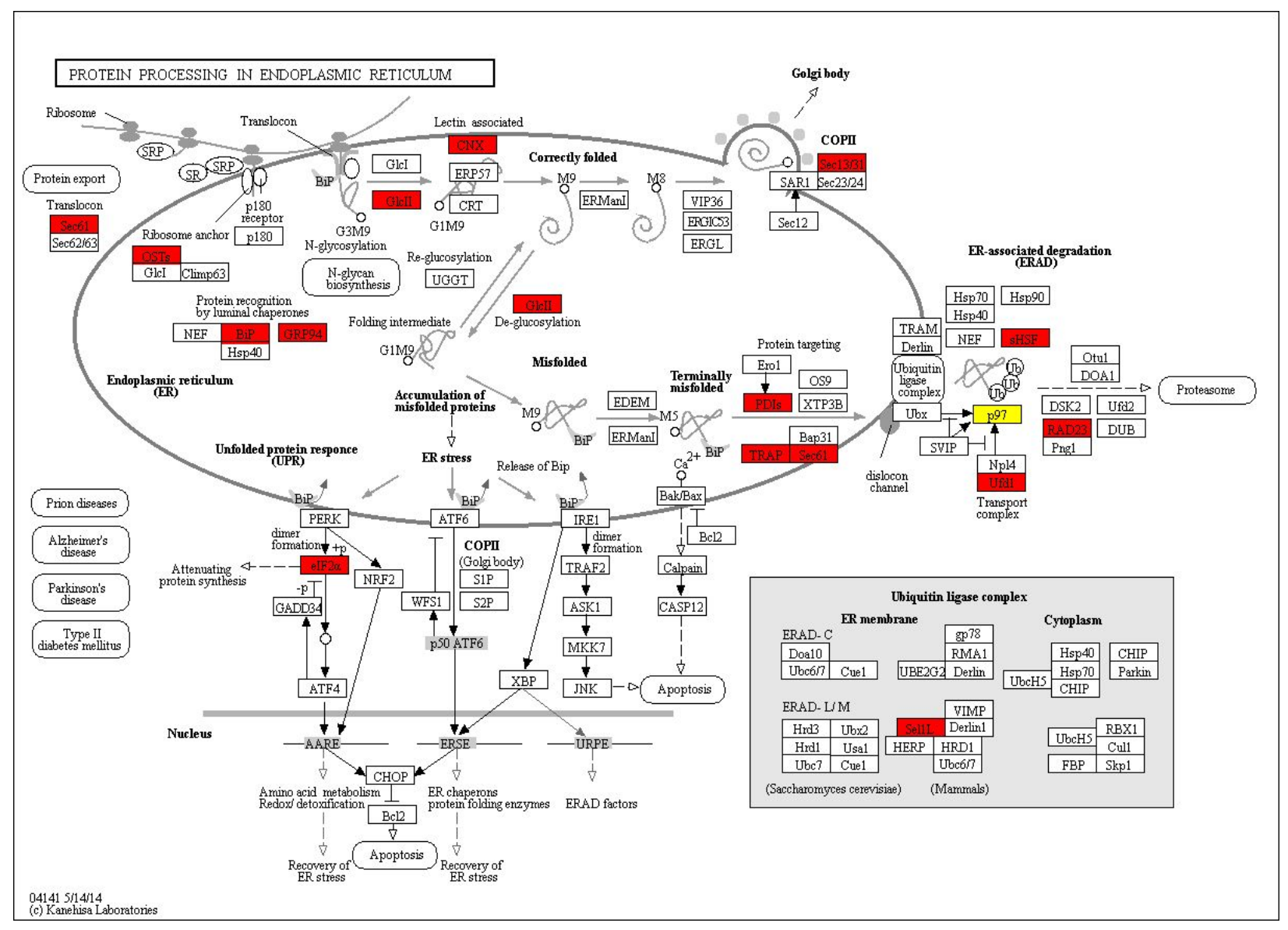

Figure S3: Mapping of ER proteins, Red indicates higher abundance and yellow indicates lower abundance. 